\title{
Why Labor Epidural Causes Fever and Why Lidocaine Burns on Injection? Role of TRPV1 Receptor in Hyperthermia: Possible Explanation of Mechanism of Hyperthermia during Labor Epidural and Burning Sensation on Injection of Local Anesthetics
}

\author{
Ilya Kozlov \\ Anesthesia Department, Wyckoff Heights Medical Center, Brooklyn, USA. \\ Email: ilyako2000@yahoo.com \\ Received June $1^{\text {st }}, 2012$; revised July $5^{\text {th }}, 2012$; accepted August $3^{\text {rd }}, 2012$
}

\begin{abstract}
The mechanisms of epidural-associated fever remain incompletely understood [1-3]. We propose that action of local anesthetic on TPRV1. The transient receptor potential cation channel subfamily V member 1 (TRPV1), also known as the capsaicin receptor and the vanilloid receptor can explain this effect and explain mechanism of burning sensation on local anesthetic injected subcutaneously or intramuscular. Role of TRPV1 receptor was not discussed previously in Obstetric Anesthesia literature. Based on available data, we propose that Local Anesthetics work as agonist/antagonist on TPRV1 receptors. Antagonist action may cause hyperthermia through modifying thermoregulation [4], agonist action may cause hyperthermia thru release of IL-6 and other mediators of inflammation [5-10]. Agonist action may explain burning sensation on injection of Local Anesthetics. Burning sensation can be diminished by increasing $\mathrm{pH}$ of Local Anesthetic solution, because vanillin receptors are stimulated by acidification through lower $\mathrm{pH}[11,12]$.
\end{abstract}

Keywords: Labor Epidural; Hyperthermia; Fever; Vanilloid Receptor; TPRV1; Lidocaine; Local Anesthetics; Neurogenic Inflammation

\section{Introduction}

\subsection{Background}

We found that to the best of our knowledge role of TRPV1 receptor was not addressed in obstetric anesthesia or obstetric reviews dedicated to the explanation of hyperthermia caused by labor epidural. We put together this review of literature to bring to attention of our colleagues, obstetric anesthesiologists and obstetricians, role of TRPV1 receptor in genesis of hyperthermia caused by labor epidural.

\subsection{Methods}

Articles in areas close to obstetric anesthesia, including pharmacology and physiology were reviewed, using PubMed service online.

\subsection{Results}

We found that acknowledging role of TRPV1 receptor offers explanation to mechanism of hyperthermia caused by labor epidural.

\section{Mechanisms Proposed in Literature}

Significant research advances were made in the last 18 months around the topic of epidural-related fever, but major gaps in knowledge persist especially with understanding the precise mechanism. The most pressing area of research is the development of well tolerated and effective prophylactic interventions to prevent maternal and fetal exposure to hyperthermia and inflammation.

Overall, $18.5 \%$ of women had a temperature more than $100.4^{\circ} \mathrm{F}$ develop during labor. However, only $4.5 \%$ of women in this low-risk population had microbiologicproven chorioamnionitis. Whereas women with epidural were far more likely to have intrapartum fever develop, $22.7 \%$ with epidural compared with $6.0 \%$ no epidural, the use of epidural analgesia was not associated with infection [13].

Elevation in maternal body temperature can be seen in a subset of laboring women in as little as 1 hour after administration of epidural analgesia. However, it is esti- 
mated that intrapartum temperature elevation usually begins 4 to 6 hours after epidural placement and rises at a rate of $0.08^{\circ} \mathrm{C}$ to $0.14^{\circ} \mathrm{C}$ per hour. Although this rise in temperature is considered idiopathic, researchers have proposed several mechanisms that might explain the phenomenon. These include an inflammatory response, oxidative stress, an alteration in sympathetic stimulation and thermoregulatory mechanisms, and subclinical chorioamnionitis.

Recent research has focused on the inflammation and oxidative stress seen in women who develop intrapartum fever. Earlier work revealed that levels of interleukin- 6 (IL-6), a proinflammatory cytokine, are elevated in women who receive epidural analgesia and become febrile. A longer duration of epidural infusion is associated with higher levels of IL-6, whereas this elevation is not seen with longer duration of labor without epidural. This suggests that the epidural itself induces an inflammatory response; the response is not a physiologic process of labor. Similar findings with additional proinflammatory mediators and reactive oxygen species seem to support this theory.

Epidural analgesia also appears to affect the body's thermoregulatory mechanisms. That epidurals cause an increase in shivering has been well-documented, but epidural analgesia also seems to be associated with a decrease in heat dissipation via sweating and hyperventilation, most likely due to a blockade of sympathetic stimulation. However, because epidurals have been reliably shown to diminish thermoregulatory capacity, it is probable that these factors contribute to the development of the associated fever.

Finally, there remains the possibility that a subclinical chorioamnionitis might underlie a subset of maternal intrapartum fevers. Women may be more likely to request an epidural if they are experiencing symptoms of subclinical chorioamnionitis, such as uterine tenderness.

Role of TRPV1 receptor was not mentioned in Obstetric Anesthesia literature known to us.

\section{Action of Local Anesthetics on TRPV1 Receptor}

$\mathrm{C}$ fibers are found in the nerves of the somatic sensory system [1]. They are afferent fibers, conveying input signals from the periphery to the central nervous system [2].

C fibres are unmyelinated unlike most other fibers in the nervous system.

C fibers are considered polymodal because they can respond to thermal, mechanical, and chemical stimuli [1]. $\mathrm{C}$ fibers respond to all kinds of physiological changes in the body. For example, they can respond to hypoxia, hy- poglycemia, hypo-osmolarity, the presence of muscle metabolic products, and even light or sensitive touch [5]. C fiber receptors include:

- C fiber nociceptors, responsible for the second, burning pain.

- C fiber warming specific receptors, responsible for warmth.

\section{Methods}

Articles found on PubMed on topics dedicated to hyperthermia caused by labor epidural and TRPV1 were reviewed and quoted.

\section{Conclusions}

The vanilloid receptor (VR-1, TRPV1) is a receptor that is found on the free nerve endings of both $\mathrm{C}$ and $\mathrm{A} \delta$ fibers that responds to elevated levels of heat $\left(>43^{\circ} \mathrm{C}\right)$ and the chemical capsaicin [14]. Capsaicin activates $\mathrm{C}$ fibers by opening a ligand-gated ion channel and causing an action potential to occur [14]. Because this receptor responds to both capsaicin and heat, chili peppers are sensed as hot [14]. VR-1 is also able to respond to extracellular acidification and can integrate simultaneous exposure to all three sensory stimuli [14]. VR-1 is essential for the inflammatory sensitization to noxious thermal stimuli. The transient receptor potential (TRP) family comprises a diverse group of cation channels that regulate a variety of intracellular signaling pathways. The TRPV1 (vanilloid 1) channel is best known for its role in nociception and sensory transmission. First studied in the dorsal root ganglia as the receptor for capsaicin, TRPV1 is now recognized to have a broader distribution and function within the central nervous system (CNS) [15]. TRPV1 regulates vasomotor tone and metabolic heat production [4]. Blocking this receptor elicits marked hyperthermia in humans [4].

Local anesthetics (LAs) block the generation and propagation of action potentials by interacting with specific sites of voltage-gated $\mathrm{Na}(+)$ channels. LAs can also excite sensory neurons and be neurotoxic through mechanisms that are as yet undefined. Nonspecific cation channels of the transient receptor potential (TRP) channel family that are predominantly expressed by nociceptive sensory neurons render these neurons sensitive to a variety of insults. It was demonstrated that the LA lidocaine activated TRP channel family receptors TRPV1 [16]. Lidocaine also induced a TRPV1-dependent release of calcitonin gene-related peptide (CGRP) from isolated skin and peripheral nerve. These data identify TRPV1 as putative key elements of LA-induced nociceptor excitation. This effect is sufficient to release CGRP, a key 
component of neurogenic inflammation, and warrants investigation into the role of TRPV1 in LA-induced neurotoxicity [16]. Stimulation of TRPV1 stimulates microglial production of IL-6, other cytokines, IL-8, Kinin B1 [4,14-17] involved in inflammation process. Lidocaine produces a short-lasting nonselective sensory and motor block in keeping with its nonselective sodium channel blocker action. However, lidocaine also acts as an agonist for the TRPV1 receptor [17].

RPV1 is also expressed at high levels in the central nervous system and has been proposed as a target for treatment not only of pain but also for other conditions such as anxiety [18]. Furthermore TRPV1 appears to mediate long term depression (LTD) in the hippocampus [19]. LTD has been linked to a decrease in the ability to make new memories, unlike its opposite long term potentiation (LTP), which aids in memory formation. A dynamic pattern of LTD and LTP occurring at many synapses provides a code for memory formation. Longterm depression and subsequent pruning of synapses with reduced activity is an important aspect of memory formation. In rat brain slices, activation of TRPV1 with heat or capsaicin induced LTD while capsazepine blocked capsaicin's ability to induce LTD [19]. In the brain stem (solitary tract nucleus), TRPV1 controls the asynchronous and spontaneous release of glutamate from unmyelinated cranial visceral afferents-release processes that are active at normal temperatures and hence quite distinct from TRPV1 responses in painful heat [20]. Hence there may be therapeutic potential in modulating TRPV1 in the central nervous system, perhaps as a treatment for epilepsy (TRPV1 is already a target in the peripheral nervous system for pain relief).

\section{Discussion}

Based on available literature, we propose that Local Anesthetics work as agonist/antagonist on TPRV1 receptors. Antagonist action may cause hyperthermia through modifying thermoregulation [4], agonist action may cause hyperthermia thru release of IL-6 and other mediators of inflammation [5,7,9,10,15,16,21]. Agonist action may explain burning sensation on injection of Local Anesthetics. Burning sensation can be diminished by increasing $\mathrm{pH}$ of Local Anesthetic solution, because vanillin receptors are stimulated by acidification through lower $\mathrm{pH}[11,12]$. We hope that this review will bring more attention of our colleagues in Obstetric anesthesia and in $\mathrm{Ob} / \mathrm{Gyn}$ to role of TRPV1 receptor, missing link between labor epidural and hyperthermia. The most interesting fact in Binshtock [17] is that TRPV1 receptor is located on sensory neurons only, not on motor neurons. It opens future possibilities to create only sensory block and avoid motor block. Another issue is confusion in defining this phenomenon of increased temperature. Should it be labeled "hyperthermia" or "fever"? Difference is important. Hyperthermia differs from fever in the mechanism that causes the elevated body temperatures: fever is caused by a change in the body's temperature set-point in hypothalamus. More studies are needed in that direction and for constructing reasonable concept of mechanism of this phenomenon.

\section{REFERENCES}

[1] S. Segal, "Labor Epidural Analgesia and Maternal Fever," Anesthesia \& Analgesia, Vol. 111, No. 6, 2010, pp. 14671475. doi:10.1213/ANE.0b013e3181f713d4

[2] L. Goetzl, "Epidural Analgesia and Maternal Fever: A Clinical and Research Update," Current Opinion in Anesthesiology, Vol. 25, No. 3, 2012, pp. 292-299. doi:10.1097/ACO.0b013e3283530d7c

[3] S. Shatken, K. Greenough and C. McPherson, "Epidural Fever and Its Implications for Mothers and Neonates: Taking the Heat," Journal of Midwifery Women's Health, Vol. 57, No. 1, 2012, pp. 82-85. doi:10.1111/j.1542-2011.2011.00105.x

[4] N. R. Gavva, J. J. Treanor, A. Garami, L. Fang, S. Surapaneni, A. Akrami, F. Alvarez, A. Bak, M. Darling, A. Gore, G. R. Jang, J. P. Kesslak, L. Ni, M. H. Norman, G. Palluconi, M. J. Rose, M. Salfi, E. Tan, A. A. Romanovsky, C. Banfield and G. Davar, "Pharmacological Blockade of the Vanilloid Receptor TRPV1 Elicits Marked Hyperthermia in Humans," Pain, Vol. 136, No. 1-2, 2008, pp. 202-210. doi:10.1016/j.pain.2008.01.024

[5] A. G. Vinuesa, R. Sancho, C. García-Limones, A. Behrens, P. Dijke, M. A. Calzado and E. Muñoz, "Vanilloid Receptor-1 Regulates Neurogenic Inflammation in Colon and Protects Mice from Colon Cancer," Cancer Research, Vol. 72, No. 7, 2012, pp. 1705-1716.

[6] N. R. Gavva, A. W. Bannon, S. Surapaneni, D. N. Hovland Jr., S. G. Lehto, A. Gore, T. Juan, H. Deng, B. Han, L. Klionsky, R. Z. Kuang, A. Le, R. Tamir, J. Wang, B. Youngblood, D. Zhu, M. H. Norman, E. Magal, J. J. S. Treanor and J.-C. Louis, "The Vanilloid Receptor TRPV1 Is Tonically Activated in Vivo and Involved in Body Temperature Regulation," Journal of Neuroscience, Vol. 27, No. 13, 2007, pp. 3366-3374.

[7] A. Leffler, A. Lattrell, S. Kronewald, F. Niedermirtl and C. Nau, "Activation of TRPA1 by Membrane Permeable Local Anesthetics," Molecular Pain, Vol. 7, 2011, p. 62.

[8] R. M. Sappington and D. J. Calkins, "Contribution of TRPV1 to Microglia-Derived IL-6 and NFkappaB Translocation with Elevated Hydrostatic Pressure," Investigative Ophthalmology \& Visual Science, Vol. 49, No. 7, 2008, pp. 3004-3017. doi:10.1167/iovs.07-1355

[9] S. Talbot, J. P. Dias1, K. Lahjouji, M. R Bogo, M. M. Campos, P. Gaudreau and R. Couture, "Activation of TRPV1 by Capsaicin Induces Functional Kinin $B_{1}$ Re- 
ceptor in Rat Spinal Cord Microglia," Journal of Neuroinflammation, Vol. 9, 2012, p. 16.

[10] C. A. Reilly, M. E. Johansen, D. L. Lanza, J. Lee, J.-O. Lim and G. S. Yost, "Calcium-Dependent and Independent Mechanisms of Capsaicin Receptor (TRPV1)-Mediated Cytokine Production and Cell Death in Human Bronchial Epithelial Cells," Journal of Biochemical and Molecular Toxicology, Vol. 19, No. 4, 2005, pp. 266-275.

[11] D. Purves, et al., "Neuroscience," Sinauer Associates Inc., Sunderland, 2004.

[12] T. A. Barnett and D. S. Kapp, "Reduction of Pain and Local Complications When Buffered Lidocaine Solution Is Used as a Local Anesthetic in Conjunction with Hyperthermia Treatments: Results of a Randomized Trial," International Journal of Radiation Oncology, Biology, Physics, Vol. 23, No. 3, 1992, pp. 585-591. doi:10.1016/0360-3016(92)90015-A

[13] L. E. Riley, A. C. Celi, A. B. Onderdonk, D. J. Roberts, L. C. Johnson, L. C. Tsen, L. Leffert, M. C. Pian-Smith, L. J. Heffner, S. T. Haas and E. S. Lieberman, "Association of Epidural-Related Fever and Noninfectious Inflammation in Term Labor," Obstetrics \& Gynecology, Vol. 117, No. 3, 2011, pp. 588-595. doi:10.1097/AOG.0b013e31820b0503

[14] M. J. Caterina, M. A. Schumacher, M. Tominaga, T. A. Rosen, J. D. Levine and D. Julius, "The Capsaicin Receptor: A Heat-Activated Ion Channel in the Pain Pathway," Nature, Vol. 389, No. 6653, 1997, pp. 816-824. doi:10.1038/39807

[15] K. W. Ho, N. J. Ward and D. J. Calkins, "Review Article TRPV1: A Stress Response Protein in the Central Nervous System," American Journal of Neurodegenerative Disease, Vol. 1, No. 1, 2012, pp. 1-14.

[16] A. Leffler, M. J. Fischer, D. Rehner, S. Kienel, K. Kistner,
S. K. Sauer, N. R. Gavva, P. W. Reeh and C. Nau1, "The Vanilloid Receptor TRPV1 Is Activated and Sensitized by Local Anesthetics in Rodent Sensory Neurons," Journal of Clinical Investigation, Vol. 118, No. 2, 2008, pp. 763-776.

[17] A. M. Binshtok, P. Gerner, S. B. Oh, M. Puopolo, S. Suzuki, D. P. Roberson, T. Herbert, C. F. Wang, D. Kim, G. Chung, A. A. Mitani, G. K. Wang, B. P. Bean and C. J. Woolf, "Coapplication of Lidocaine and the Permanently Charged Sodium Channel Blocker QX-314 Produces a Long-Lasting Nociceptive Blockade in Rodents," Anesthesiology, Vol. 111, No. 1, 2009, pp. 127-137.

[18] V. Di Marzo, K. Starowicz and L. Cristino, "TRPV1 Receptors in the Central Nervous System: Potential for Previously Unforeseen Therapeutic Applications," Current Pharmaceutical Design, Vol. 14, No. 1, 2008, pp. 42-54. doi: $10.2174 / 138161208783330790$

[19] H. E. Gibson, J. G. Edwards, R. S. Page, M. J. Van Hook and J. A. Kauer, "TRPV1 Channels Mediate Long-Term Depression at Synapses on Hippocampal Interneurons," Neuron, Vol. 57, No. 5, 2008, pp. 746-759. doi:10.1016/j.neuron.2007.12.027

[20] J. H. Peters, S. J. McDougall, J. A. Fawley, S. M. Smith and M. C. Andresen, "Primary Afferent Activation of Thermosensitive TRPV1 Triggers Asynchronous Glutamate Release at Central Neurons," Neuron, Vol. 65, No. 5, 2010, pp. 657-669. doi:10.1016/j.neuron.2010.02.017

[21] Z. Wang, Y. Yang, H. Yang, J. E. Capó-Aponte, S. D. Tachado, J. M. Wolosin and P. S. Reinach, "NF-kB Feedback Control of JNK1 Activation Modulates TRPV1-Induced Increases in IL-6 and IL-8 Release by Human Corneal Epithelial Cells," Molecular Vision, Vol. 17, 2011, pp. 3137-3146. 